OPEN ACCESS

Edited by:

Stephen D. Ginsberg, Nathan Kline Institute for Psychiatric Research, United States

Reviewed by:

Susheel Vijayraghavan, Western University (Canada), Canada Amy F. T. Arnsten, Yale University, United States

*Correspondence: Tara L. Moore tImoore@bu.edu

Received: 12 August 2021 Accepted: 23 October 2021 Published: 26 November 2021

Citation:

Moore $T L$, Young $D A$, Killiany $R J$, Fonseca $K R$, Volfson D, Gray DL, Balice-Gordon R and Kozak R (2021) The Effects of a Novel Non-catechol Dopamine Partial Agonist on Working Memory in the Aged Rhesus Monkey.

Front. Aging Neurosci. 13:757850

doi: 10.3389/fnagi.2021.757850

\section{The Effects of a Novel Non-catechol Dopamine Partial Agonist on Working Memory in the Aged Rhesus Monkey}

\author{
Tara L. Moore ${ }^{1,2 *}$, Damon A. Young ${ }^{3}$, Ronald J. Killiany ${ }^{1,2}$, Kari R. Fonseca ${ }^{4}$, \\ Dmitri Volfson ${ }^{3}$, David L. Gray ${ }^{3}$, Rita Balice-Gordon ${ }^{3}$ and Rouba Kozak ${ }^{3}$
}

${ }^{1}$ Department of Anatomy \& Neurobiology, Boston University School of Medicine, Boston, MA, United States, ${ }^{2}$ Center for Systems Neuroscience, Boston University, Boston, MA, United States, ${ }^{3}$ Internal Medicine Research Unit Pfizer Worldwide Research, Development and Medical Pfizer Inc., Cambridge, MA, United States, ${ }^{4}$ Medicine Design, Pfizer Worldwide Research, Development and Medical Pfizer Inc., Cambridge, MA, United States

Aged-related declines in cognition, especially working memory and executive function, begin in middle-age and these abilities are known to be mediated by the prefrontal cortex (PFC) and more specifically the dopamine (DA) system within the PFC. In both humans and monkeys, there is significant evidence that the PFC is the first cortical region to change with age and the PFC appears to be particularly vulnerable to age-related loss of dopamine (DA). Therefore, the DA system is a strong candidate for therapeutic intervention to slow or reverse age related declines in cognition. In the present study, we administered a novel selective, potent, non-catechol DA D1 R agonist PF-6294 (Pfizer, Inc.) to aged female rhesus monkeys and assessed their performance on two benchmark tasks of working memory - the Delayed Non-match to Sample Task (DNMS) and Delayed Recognition Span Task (DRST). The DNMS task was administered first with the standard $10 \mathrm{~s}$ delay and then with 5 min delays, with and without distractors. The DRST was administered each day with four trials with unique sequences and one trial of a repeated sequence to assess evidence learning and retention. Overall, there was no significant effect of drug on performance on any aspect of the DNMS task. In contrast, we demonstrated that a middle range dose of PF-6294 significantly increased memory span on the DRST on the first and last days of testing and by the last day of testing the increased memory span was driven by the performance on the repeated trials.

Keywords: dopamine, aging, prefrontal cortex, rhesus monkey, memory

\section{INTRODUCTION}

Age-related decline in working memory and executive function have been well documented in both humans and monkeys (Moore et al., 2003, 2006; Alexander et al., 2012; Bizon et al., 2012; Murman, 2015; Salthouse, 2016). Working memory and executive function are mediated by the prefrontal cortex (PFC) and more specifically through the normal function of the dopamine (DA) 
system within the PFC (Mishkin and Pribram, 1956; Mishkin, 1957; Rosvold et al., 1961; Sawaguchi and Goldman-Rakic, 1991; De Bundel et al., 2013; Takahashi, 2013; Wass et al., 2013; Arnsten et al., 2015; Burke et al., 2018; Wang et al., 2019; Soutschek et al., 2020a,b). There is a significant decrease in DA levels with age (Sawaguchi et al., 1990; Sawaguchi and Goldman-Rakic, 1991; Arnsten et al., 1994; Thierry et al., 1998; Volkow et al., 1998, 2000) and the PFC appears to be particularly vulnerable to age-related loss of dopamine with a $56 \%$ decrease in DA neurotransmitter concentration in monkeys 18 years and older (Goldman-Rakic and Brown, 1981; Arnsten et al., 1994). Numerous studies have also demonstrated age-related changes in DA receptors (Brozoski et al., 1979; Arnsten et al., 1994, 1995; Volkow et al., 1996, 1998, 2000; Cai and Arnsten, 1997; Wang et al., 1998; Moore et al., 2005; Bäckman et al., 2006, 2010; Morcom et al., 2010). Evidence from PET scans have shown an age-related decline in DA receptor densities in the striatum and frontal cortex of the aging brain (Bäckman et al., 2011; Karlsson et al., 2011; Rieckmann et al., 2011) and concentrations of D1 receptors are related to performance on tasks of working memory and executive function in middle-age and aged humans and monkeys (Arnsten et al., 1994, 1995; Cai and Arnsten, 1997; Müller et al., 1998; Castner and Goldman-Rakic, 2004; Moore et al., 2005; Bäckman et al., 2011; Rieckmann et al., 2011). Further, it has been demonstrated that administration of DA agonists to aged monkeys improves working memory (Cai and Arnsten, 1997; Castner and Goldman-Rakic, 2004) while administration of DA antagonists decreases performance on working memory tasks (Fischer et al., 2010).

Based on the studies described above, it seems likely that age associated deficits in cognition may be related to loss of DA signaling and therefore this system is a strong candidate for therapeutic intervention to slow or reverse these declines. For example, the administration of a DA D1 receptor antagonist, ecopipam, altered performance in aged rodents on choice tasks and their performance was partially enhanced by coadministration of D1 agonists (Yohn et al., 2015). Recently, Wang et al. (2019), demonstrated that the DA D1 agonist PF-3628, which has a moderate affinity for the DA D1 receptor, when applied directly to DLPFC neurons in aged monkeys performing a delay dependent working memory task caused enhanced task related firing. Building on these promising findings, in the present study, we administered a novel selective, potent, non-catechol DA D1 R partial agonist PF-6294 (Pfizer, Inc.) to aged female rhesus monkeys and assessed their performance on two benchmark tasks of working memory. PF-6294 is structurally very similar to other D1 partial agonists recently described by Pfizer (Gray et al., 2018; Wang et al., 2019; Kozak et al., 2020; Young et al., 2020). This compound was chosen based on its suitable non-human primate pharmacokinetics and its D1 pharmacology, including affinity for human $\mathrm{D} 1$ receptors $(\mathrm{Ki}=17 \mathrm{nM})$, functional partial agonism at human D1 receptors (EC50 $=36 \mathrm{nM}$, Intrinsic activity $=69 \%$ relative to dopamine), and in particular, binding to the nonhuman primate $\mathrm{D} 1$ receptor $(\mathrm{Ki}=14 \mathrm{nM})$. We designed the experiment to assess both acute and repeat dose effects on two different tasks to report on subcomponents of working memory performance. We also tested a range of doses with an overall goal of building on our understanding of the role of D1 receptor activation in specific aspects of the working memory process.

\section{MATERIALS AND METHODS}

\section{Subjects}

Eight, behaviorally naive, middle aged-old (17-23 yrs of age; equivalent to approximately 51-69 human years), female rhesus monkeys ( $M$. mulatta) were used in the present study. All of the monkeys were obtained from national primate research facilities or private vendors and had known birth dates and complete health records. Before entering the study, monkeys received complete medical examinations. In addition, all monkeys underwent magnetic resonance imaging (MRI) to ensure there was no occult neurological damage prior to the start of the study. Results of the medical exams and MRIs revealed that all monkeys were healthy at the time of the study. While on study, monkeys were individually housed in colony rooms where they were in constant auditory and visual range of other monkeys in the Animal Science Center (ASC) of Boston University School of Medicine. This facility is fully AAALAC accredited and all research protocols were approved by the Boston University Institutional Animal Care and Use Committee.

Diet consisted of Purina Monkey Chow (Purina Mills Inc, St. Louis, MO, United States) supplemented by fruit, vegetables and forage with feeding taking place once per day, immediately following behavioral testing. Water was available continuously. The monkeys were housed under a 12-h light/dark cycle with cycle changes occurring in a graded fashion over the course of an hour. Following a mandatory 6-week quarantine period and acclimation to the colony room, animals were ready to begin pre-training on cognitive tasks.

\section{Binding to D1 in Non-human Primates}

PF-6294 and other test compounds were synthesized as free bases by Pfizer worldwide medicinal chemistry. For PF-6294, batch number 0001 was tested. All compounds were dissolved in 100 percent DSMO and stored in a Nitrogen environment. Human D1 binding affinity and Human cAMP functional activity were determined according to a previously described method (Gray et al., 2018). D1 binding studies in non-human primate striatal tissue were conducted as follows.

Compounds were titrated to assess binding as a 10 point concentration response. Radioligand was purchased from Perkin Elmer (Boston, MA, United States). Non-specific binding controls were purchased commercially. Primary striatal tissue from a cynomolgus monkey brain was used for receptor binding studies. The radioligand was $[3 \mathrm{H}]-\mathrm{SCH} 23390(1.0 \mathrm{nM})$ and the tissue concentration was $9 \mathrm{mg} / \mathrm{mL}$. The $\mathrm{Kd}$ of the radioligands used for these binding experiments was $1.7 \mathrm{nM}$, determined in separate saturation experiments.

Tissue preparation buffer was $50 \mathrm{mM}$ Tris buffer containing $2.0 \mathrm{mM} \mathrm{MgCl} 2$ at $\mathrm{pH} 7.4$ (at $4^{\circ} \mathrm{C}$ ). Monkey striatal tissue was homogenized in tissue buffer using a Polytron (setting 5) and centrifuged at 40,000 $\mathrm{g}$ for $10 \mathrm{~min}$. Tissue was re-suspended in tissue preparation buffer using a polytron and centrifuged for 
$10 \mathrm{~min}$ at $40,000 \mathrm{~g}$. The resulting pellet was then re-suspended in $50 \mathrm{mM}$ Tris $\mathrm{HCl}$ with $4 \mathrm{mM} \mathrm{MgSO} 4$ and $0.5 \mathrm{mM}$ EDTA assay buffer at $\mathrm{pH} 7.4$ at room temperature.

Test compounds (including PF-6294), (+)-Butaclamol as a non-specific control, and DMSO controls $(2.5 \mu \mathrm{L})$ were added to 96-well plates (Costar 3363) at 100-fold their final concentrations, followed by $50 \mu \mathrm{L}$ radioligand at 5 -fold the final concentration. The exact concentration of radioligand for each experiment was determined by liquid scintillation counting. Competition binding was initiated by the addition of $200 \mu \mathrm{L}$ of tissue homogenate and incubated for $30 \mathrm{~min}$ at $37^{\circ} \mathrm{C}$. After incubation, assay samples were rapidly filtered through Unifilter-96 GF/B PEIcoated filter plates (Perkin Elmer, Boston, MA, United States) using a plate harvester (Packard) and rinsed with ice-cold $50 \mathrm{mM}$ Tris buffer ( $\mathrm{pH} 7.4$ at $4^{\circ} \mathrm{C}$ ). Filter plates were dried overnight at room temperature. Plates were bottom sealed prior to the addition of $50 \mu \mathrm{L} /$ well Ecolume (MP Biomedicals, Solon, $\mathrm{OH}$, United States) scintillation fluid. Plates were then top sealed and read on a Trilux (Perkin Elmer, Boston, MA, United States). Percent effects for each concentration were determined, and IC50 values were calculated using a logistic 4 parameter fit model. Ki values were calculated from the IC50 values using the ChengPrusoff equation: $\mathrm{Ki}=\mathrm{IC} 50 /(1+([\mathrm{L}] / \mathrm{Kd}))$, where $\mathrm{L}$ is the concentration of the radioligand used in the experiment; $\mathrm{Kd}$ is the affinity of the radioligand (determined in separate saturation experiments). Additional proprietary and reference compounds were also included in each run to confirm assay consistency.

\section{Drug/Vehicle Administration}

Three doses of drug $(0.002,0.004$, and $0.012 \mathrm{mg} / \mathrm{kg}$ ) were used in the present study. The doses for the current study were selected based on initial subcutaneous pharmacokinetic study data showing linear PK across a similar dose range following $\mathrm{SC}$ administration (data is not reported). At the chosen doses the exposure was predicted to cover a wide range of D1 receptor occupancy.

Drug was dissolved in 5\% dimethyl sulfoxide $+5 \%$ Cremophor $\mathrm{EL}+90 \%$ sterile water or sterile saline +0 to 3 molar equivalents of hydrochloric acid to a $\mathrm{pH} \sim 3-4$ for subcutaneous administration. Total administration volume ranged from 2 to $10 \mathrm{ml}$ depending on the weight of the individual monkey.

The study was divided into three drug/testing sessions and each monkey received daily administration of either one of the three doses (labeled A, B or C) or the vehicle (labeled D) only during each of the three sessions. A balanced cross-over design was used and each monkey received two of the three dose levels of PF-6294 and 1 dose of vehicle across the three testing periods (see Table 1). The doses administered were randomly assigned to each monkey. Drug or vehicle were administered by subcutaneous injection in the thigh and injection sites were alternated between the left and right thighs and distributed across each thigh to prevent irritation. Drug was administrated once each day, one hour prior to beginning the daily testing session. Each drug/testing session spanned three weeks and within each session, monkeys were dosed every day for 22 consecutive days and were tested five days a week (Monday-Friday). A six-week wash-out period occurred between each drug/testing session
TABLE 1 | Balanced cross-over design for drug administration across testing periods.

\begin{tabular}{cccc}
\hline Cohort & $\begin{array}{c}\text { Testing/drug } \\
\text { administration } \\
\# \mathbf{1}\end{array}$ & $\begin{array}{c}\text { Testing/drug } \\
\text { administration } \\
\text { 2 }\end{array}$ & $\begin{array}{c}\text { Testing/drug } \\
\text { administration } \\
\# 3\end{array}$ \\
\hline $1(n=3)$ & D & B & C \\
$2(n=2)$ & A & D & C \\
$3(n=3)$ & A & B & D \\
\hline
\end{tabular}

during which no drug was administered and monkeys were not tested. All personnel were blind to treatment groups.

Baseline blood samples were collected prior to entering the study and then additional blood samples were drawn at two time points during each drug/testing session. First, blood was drawn the day after the completion of a testing session. For this blood draw, the drug or vehicle was administered the morning following the last day of testing in a session (Day 22 of dosing) and then blood was drawn one hour later. Second, blood was drawn on the last day of each wash-out period. Blood samples were collected into vacutainers containing K3EDTA and maintained on ice prior to centrifugation. Following centrifugation, the resultant plasma samples were transferred to polypropylene tubes and stored frozen at $-80^{\circ} \mathrm{C}$ until analysis of compound concentrations.

\section{Plasma Protein Binding}

The unbound fraction of PF-6294 in monkey plasma (fu,p) was determined by equilibrium dialysis using standard procedures (Di et al., 2017).

\section{Calculation of Receptor Occupancy}

The unbound plasma concentrations (Cp,u) of PF-6294 were calculated by multiplying the total plasma concentrations $\left(C_{p}\right)$ by the fraction unbound in plasma $\left(f_{u, p}\right)$. The unbound concentrations in the plasma were presumed to be in equilibrium with the unbound brain concentrations. The receptor occupancy (RO) of PF-6294 was calculated based upon the $\mathrm{C}_{\mathrm{p}, \mathrm{u}}$ and the monkey in vitro $\mathrm{K}_{i}(14 \mathrm{nM})$ determined through use of a radioligand binding assay in monkey striatal tissue through use of the following equation:

$$
\mathrm{RO}=\frac{C_{p, u}}{\left(C_{p, u}+K_{i}\right)} \times 100
$$

\section{Quantitation of Compound in Biological Samples}

A non-validated liquid chromatography tandem massspectrometry (LC-MS/MS) method was used for the quantitation of PF-6294 in monkey plasma and plasma protein binding samples. All samples were subjected to protein precipitation followed by LC-MS/MS analysis to determine PF-6294 concentrations.

\section{Cognitive Testing}

All monkeys were initially familiarized with cognitive testing in a Wisconsin General Testing Apparatus (WGTA). All monkeys 


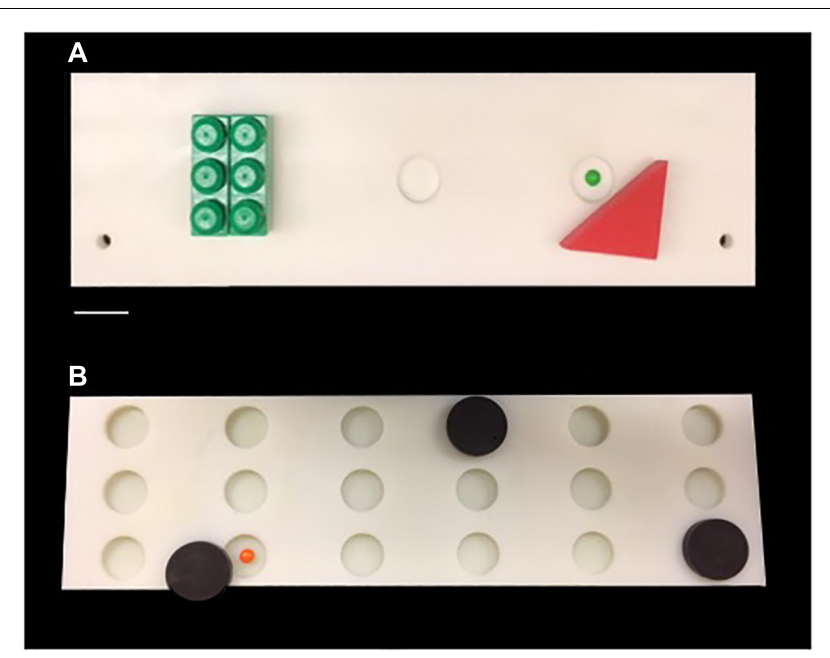

FIGURE 1 | (A) Photograph of the testing board used for Delayed Non-match Sample Task. (B) Photograph of the testing board used for Delayed Recognition Span Task. Scale Bar $=5.0 \mathrm{~cm}$.

were trained only to displace a single gray plaque placed pseudorandomly over one of three lateral food wells in a wooden testing board to obtain a reward. Cut grapes, raisins and/or small pieces of candy were used as rewards during testing. Monkeys were trained until they responded for 40 consecutive trials on two successive days.

\section{Baseline Testing}

Monkeys were then trained on the Delayed Non-matching to Sample task (DNMS). The DNMS task is a benchmark task of recognition memory that is widely used with non-human primates (Figure 1A). The DNMS task assesses the monkey's ability to identify a novel from a familiar stimulus. Each trial of DNMS begins with a sample object presented over the central baited food well of the same wooden testing board used during testing familiarization. The monkey was permitted to displace the object and obtain the food reward. The door was then lowered, and the original, now familiar, sample object was placed over an unbaited lateral well and a new, novel, unfamiliar object is placed over the other lateral well that was baited. Ten seconds after the original sample trial, this choice trial was begun and the monkey must choose the unfamiliar, novel object in order to obtain the reward. Twenty seconds later, the next trial was initiated with a new, novel sample object presented over the baited central well followed $10 \mathrm{~s}$ later by another recognition trial using that second sample object and another new novel object. The position of the two objects on successive recognition trials was varied from left to right lateral wells in a predetermined pseudorandom order. Monkeys were trained on this task until their performance reached a criterion of $90 \%$ correct responses over 100 trials.

Following reaching criterion on the DNMS task, monkeys then completed 5 days (5 trials per day) of the Delayed Recognition Span Task (DRST)-spatial. The DRST-spatial task requires the monkey to identify, trial by trial, a new stimulus among an increasing array of serially presented, familiar stimuli
(Figure 1B). Fifteen identical plain brown discs $(6 \mathrm{~cm}$ in diameter) were used as stimuli. For the first sequence, one disc was placed over one of the eighteen wells, which was baited. The screen was raised and the monkey is allowed to displace the disc to obtain the reward. The screen was lowered and a second disc is placed on the board over a baited well while the first disc was returned to its original position over the now unbaited well. After $10 \mathrm{~s}$, the screen was raised and the monkey was required to identify and displace the new disc that was in a novel spatial location in order to obtain the reward. Each successive correct response trial was followed by the addition of a new disc on the next trial until the monkey made an error (i.e., chooses a previously chosen disc) or reaches a span of 9 correct responses. With the occurrence of the first error, the trial was terminated and the number of discs on the test tray minus one constitutes the recognition span score for that trial (i.e., number of correct responses). One trial each day was a single repeated sequence that was included in the test administration based on Hebb's Repetition Paradigm that describes that the serial recall of a repeated sequence of information or stimuli gradually develops into stable, durable and longer-term memory (Cohen and Johansson, 1967; Szmalec et al., 2009). Therefore, quantifying the span on repeated and non-repeated trials allows us to determine if there is evidence of greater learning and retention of a repeated sequence even with in the presence of an impairment in spatial working memory on the non-repeated trials. The total span was calculated by taking the mean total span across the five days. The total repeated span was calculated by taking the mean of the span achieved on the repeated sequence each day. The total non-repeated span is calculated by subtracting the repeated span from the total span.

\section{Testing During Drug/Test Sessions}

During each drug/test session, we administered three tasks: DNMS Basic, DNMS delays (with and without interference) and Delayed Recognition Span Task (DRST).

\section{Delayed Non-matching-to-Sample (DNMS - Basic Acquisition)}

During the 1st week of testing in each testing session, the DNMS task was administered as described above for 20 trials per day for five days. The total errors made by the monkey during the 100 trials was recorded (Table 2).

\section{Delayed Non-matching-to-Sample Delay (DNMS - 5-min Delay)}

During the 2nd week of each drug/test session, a DNMS delay task was administered. DNMS delays utilizes the same concept of the DNMS basic task, but instead of a 10-s delay between presentation of the sample and choice objects there was a 5-min delay after the sample object is initially presented and before the choice trial. Ten trials were administered with the first 5 trials administered only with 5-min delays and the last five trials also included an "interference" component. The interference component involved administering one trial of a simple color discrimination task to the monkey during each of the last five 


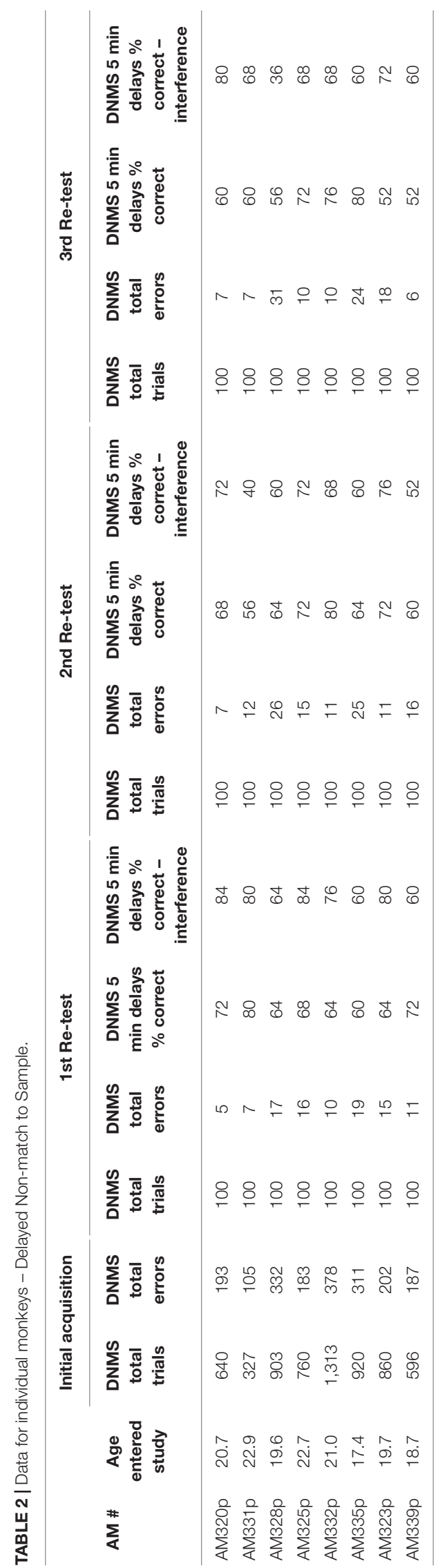

trials within the 5-min delay. Specifically, 30-s after the initial sample object was presented to the monkey, green and red plaques were placed over the left and right wells (out of sight of the monkey) and the monkey had to choose one of the plaques. For all monkeys the "red" plaque was always correct on all trials. Then at the end of the 5-min delay, the choice trial in the original DNMS delay trial was administered. The total number of correct responses during the non-interference and the interference trials were recorded for each monkey (Table 2).

\section{Delayed Recognition Span Task-Spatial}

During the 3rd week of each drug/test session, the monkeys completed the DRST-spatial task. Five trials were presented each day for five consecutive days (25 trials) during each test session. One trial each day was a single sequence that was repeated each day ( 5 trials). The total span was calculated by taking the mean total span across the five days. The total repeated span was calculated by taking the mean of the span achieved on the repeated sequence each day. The total non-repeated span is calculated by subtracting the repeated span from the total span (Table 3).

\section{Data Analysis}

Data were analyzed using $\mathrm{R} \quad 3.2 .3$ statistical software. Performance on basic and delayed with and without interference tasks were used as endpoints for DNMS. Total span, non-repeat span and repeat span were used as endpoints for the DRST. Performance on the DRST and DNMS tasks was analyzed using repeated measures mixed model ANOVA. The effects of baseline, period, treatment, day, and treatment by day interaction were included as fixed factors, while monkey and monkey by treatment interaction served as random factors. The model was fitted using the method of restricted maximum likelihood, and significance of fixed effects was evaluated using Kenward-Roger degrees of freedom estimation. Significant ANOVA results were followed by Dunnett's post-hoc comparisons against control group, separately at each time point. Data were considered statistically significant at a level of $p<0.05$.

\section{RESULTS}

\section{Plasma Protein Binding}

The fraction unbound of PF-6294 in monkey plasma was 0.178.

\section{Plasma Concentrations of PF-6294 in Monkey Plasma}

The mean plasma concentrations of PF-6294 following SC administration at $0.002,0.004$, and $0.012 \mathrm{mg} / \mathrm{kg}$ were $1.00,3.96$, and $6.50 \mathrm{ng} / \mathrm{mL}$, respectively, at $1 \mathrm{~h}$ post-dose. Conversion of these concentrations to unbound molar concentrations resulted in unbound plasma concentrations of $0.48,1.89$, and $3.10 \mathrm{nM}$, respectively ( $\mathrm{MW}=373.35 \mathrm{~g} / \mathrm{mol}$ ).

\section{Receptor Occupancy at $\mathbf{1} \mathrm{h}$ Post-dose}

At $1 \mathrm{~h}$ post-dose, the calculated D1 receptor occupancies were $3.3,12$, and $18 \%$ at the dose levels of $0.002,0.04$, 


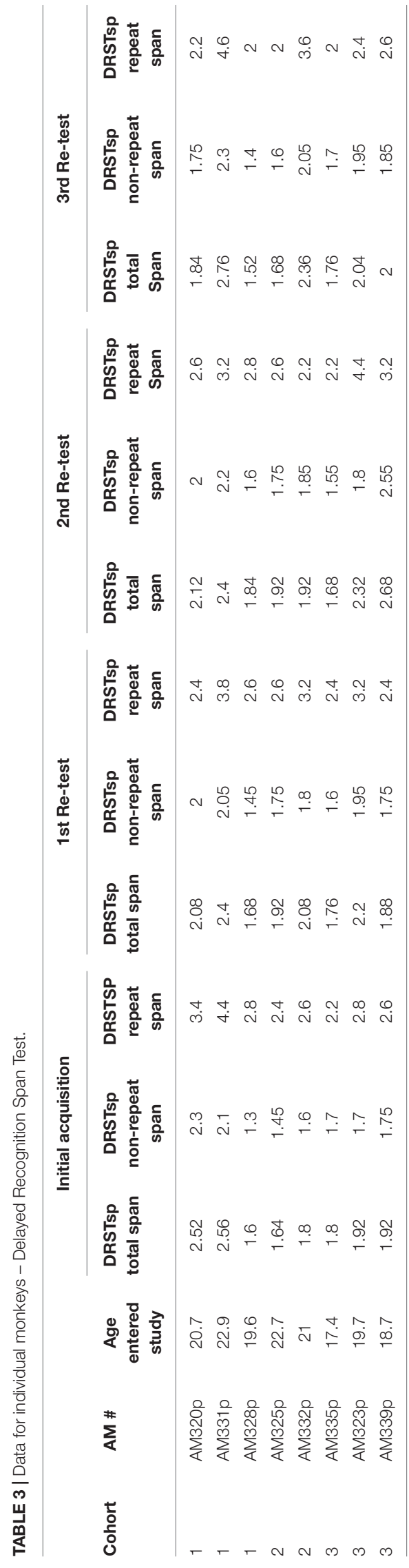

and $0.12 \mathrm{mg} / \mathrm{kg}$, respectively. The use of unbound plasma concentrations in the calculation presumes there is no hindrance to the distribution of PF-6294 across the blood-brain barrier and that unbound brain concentrations are in equilibrium with unbound plasma concentrations.

\section{Delayed Non-matching to Sample - Basic Task}

For the basic version of the DNMS task, there were no significant effects on performance at any dose of PF-6294 on any individual day of testing (Figure 2A). When the data was collapsed across days, there was still no significant effect of drug at any dosing (Figure 2B). The monkeys were performing the task at $>80 \%$ correct at baseline, in line with historical baseline data for similar tasks, and the presence of D1 agonist did not alter the number of correct selections. Even

\section{Delayed Non-matching to Sample - Delay}

Similar to the lack of effect of treatment on performance in the basic DNMS task, no dose of PF-6294 had an effect on the task with increased delays (Figure 2C). The introduction of interference also yielded no significant changes in performance at any dose (Figure 2D).

\section{Delayed Recognition Span Task-Spatial}

For the total trials of the DRST, there were significant main effects on performance from baseline $\left[F_{(1,5.992)}=6.1216, p=0.048\right]$ and treatment $\left[F_{(3,12.552)}=4.3605, p=0.026\right]$, as well as an interaction between treatment and day $\left[F_{(16,80)}=3.1608\right.$, $p=0.0003]$. Post hoc analysis revealed a significantly higher span, as compared to baseline, for the middle dose on day 1 $\left[t_{(118.51)}=4.164, p=0.0002\right]$, as well as on day $5\left[t_{(118.51)}=2.531\right.$, $p=0.035]$ (Figure 3A). The total span was also higher on day 4 for the highest dose $\left[t_{(118.21)}=2.659, p=0.025\right.$; see Figure 3A]. Overall, when collapsed by day, a significantly higher total span was achieved at the middle dose $(0.004 \mathrm{mg} / \mathrm{kg})$ $\left[t_{(117.01)}=3.379, p=0.003\right.$; see Figure 3B $]$. This increase was due to the effect on repeated trials $\left[t_{(96.36)}=3.297, p=0.004\right]$, but not non-repeated trials (see Figures 3C,D). While there was not a significant difference between repeated and nonrepeated spans, performance on the repeated span by treated monkeys did improve across the testing sessions suggesting that although the trial was only repeated 4 times after the initial presentation, there is evidence of enhanced learning of the stimuli over repeated trials.

For repeated trials only, there was a significant main effect from baseline $\left[F_{(1,6.542)}=7.0399, p=0.035\right]$ and an interaction between treatment and day $[F(16,80)=3.468, p=0.0001]$. For non-repeated trials, there were no significant main effects or interactions.

The increase in total span on day 1 at the middle dose was driven by increases on both repeated $[t(119.96)=2.482$, $p=0.040]$ and non-repeated trials $[t(120)=2.759, p=0.019$; see Figures $\mathbf{4 A - C}$ ]. It is not surprising that there was an equal effect of the repeated and non-repeated trials at Day 1 since 

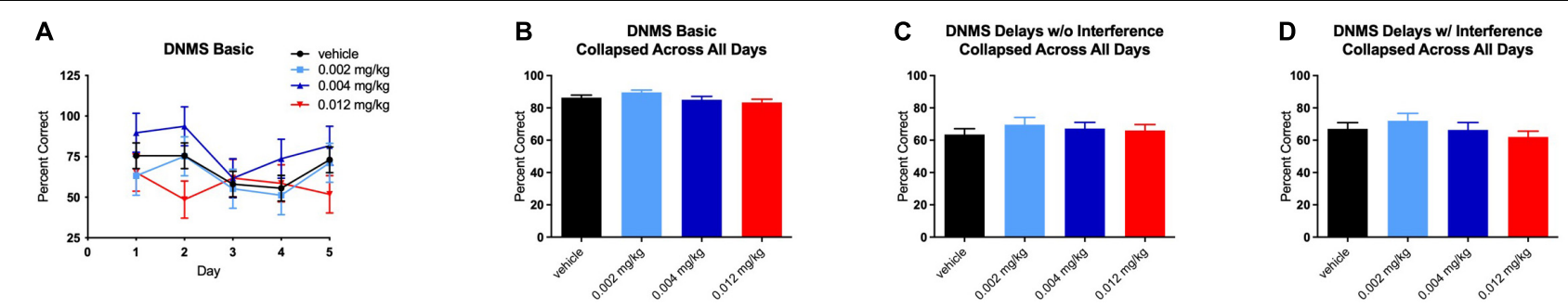

FIGURE 2 | Effect of PF-6294 on performance on the basic DNMS task (A) on individual testing days at each dose (B) collapsed across all days and (C) on the delayed version without interference and (D) with interference. No dose had a significant effect on performance on any version of the task. Error bars = SEM.
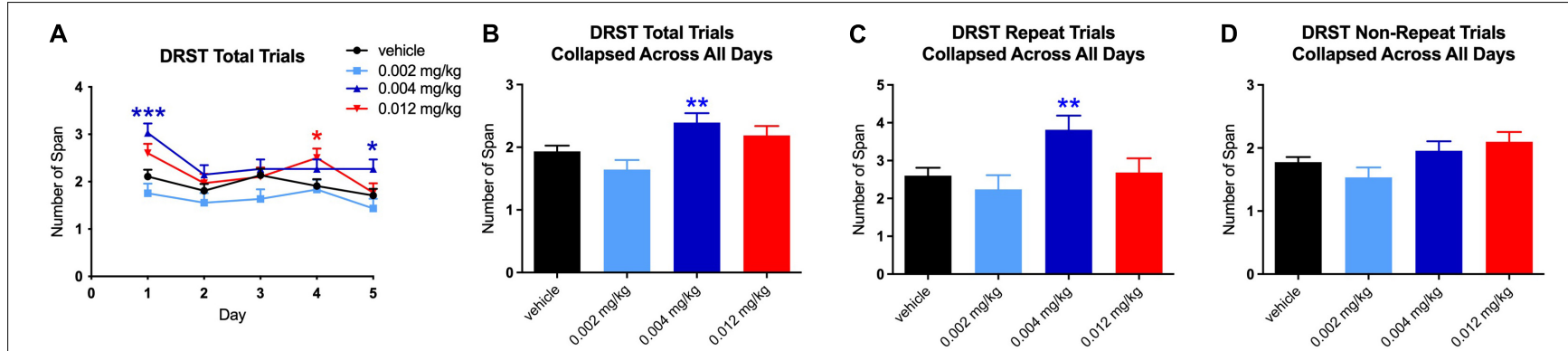

FIGURE 3 | Effect of PF-6294 on DRST Spatial Overall Performance. (A) Analysis revealed across all days for total trials of the DRST a significantly higher span for the middle dose on day $1\left(^{\star \star \star} p=0.0002\right)$ and on day $5\left({ }^{*} p=0.035\right)$. The total span was also higher on day 4 for the highest dose (red $\left.{ }^{\star} p=0.025\right)$. (B) Overall, when collapsed by day, there was a significantly higher total span achieved at the middle dose $\left({ }^{* *} p=0.003\right)$. (C,D) Graphs show that the increase was due to the effect on repeated trials $(p=0.004)$, but not non-repeated trials. Error bars $=$ SEM.

this is the 1st time the repeated trial was administered and therefore was not qualitatively different from the non-repeat trials. However, the increase in total span on day 5 for the middle dose was seen only on repeated trials $[t(119.26)=4.442, p=0.0001$; see Figures $\mathbf{5 A}-\mathbf{C}$ ]. Despite the highest dose producing an increase in total span on day 4 , when tested separately, neither repeated nor non-repeated trials on that day were significantly different from vehicle.

\section{DISCUSSION}

In the current study, we demonstrated that there was a significant effect of a novel selective, potent, non-catechol D1R agonist (PF-6294, Pfizer, Inc.) on a task of spatial working memory (DRST). Specifically, the middle range dose resulted in a significantly higher total memory span when compared to vehicle. These findings are of particular interest given that the DRST relies on the PFC for normal performance (unpublished data - dorsolateral prefrontal cortex lesions in young monkeys significantly impairs performance on DRST) and this is a cortical area that shows age-related changes in DA levels. Further, PF6294 showed a non-linear dose response on the DRST, a pattern which has been reported previously in studies examining the impact of D1 agonist pharmacology on preclinical cognitive tasks (Arnsten et al., 1994, 2015; Cai and Arnsten, 1997; Wang et al., 2019).

In contrast, there was no effect on performance on the DNMS task (basic acquisition or delay condition). However, unlike the
DRST, the DNMS task, in particular the re-acquisition and delay phases of the task, are considered to rely more on the medial temporal lobe (Rapp and Amaral, 1989, 1991; Beason-Held et al., 1999). Further, as the DRST task was the 3rd task administered in each testing session, the unique effect on the performance of this task may be the result of the cumulative dosing that occurred prior to administration of the DRST task. Future studies with counterbalancing of task administration (eg. administration of DRST at week 1 and DNMS at week 3) may provide additional insight into the effect of DA agonists on DNMS performance.

\section{Cognition and the Prefrontal Cortex}

It is well established that normal aging is characterized by declines in short-term memory, psychomotoric speed, naming, and executive function (Albert, 1993; Bachevalier, 1993; Arnsten et al., 1994; Arnsten and Jentsch, 1997; Herndon et al., 1997; Cotman et al., 2002; Moore et al., 2003, 2005, 2006; Fisk and Sharp, 2004; Drag and Bieliauskas, 2010; Alexander et al., 2012; Darusman et al., 2014) in both humans and monkeys. Of these cognitive abilities, executive function (EF) is one of earliest cognitive domains to evidence change in humans (Fisk and Sharp, 2004; Finch, 2009; Drag and Bieliauskas, 2010) and nonhuman primates (Bachevalier, 1993; Lai et al., 1995; Herndon et al., 1997; Moore et al., 2003, 2006; Darusman et al., 2014). Spatial working memory, a component of EF, is mediated in part by the prefrontal cortex (PFC) (Raz et al., 1997; Moore et al., 2009; McEwen and Morrison, 2013; Borella et al., 2014; Toepper et al., 2014; Arshad et al., 2016; Johnson et al., 2016; Kwon et al., 2016; 


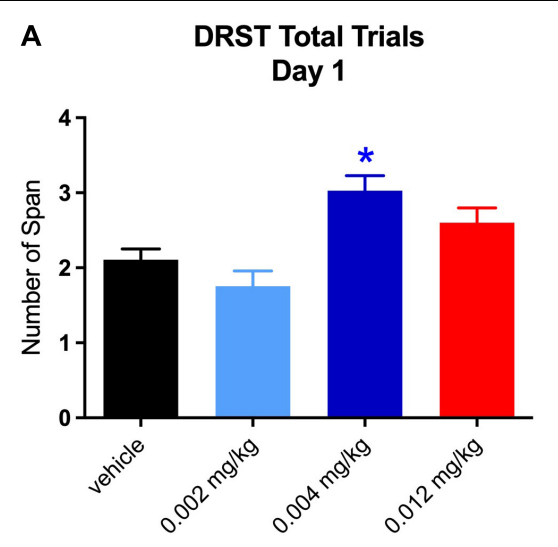

\section{B}

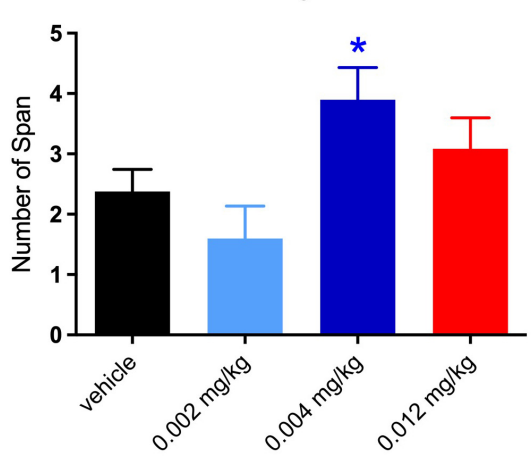

C
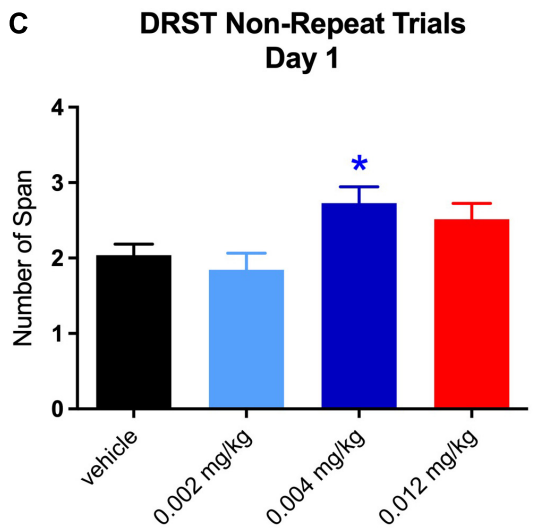

FIGURE 4 | Effect of PF-6294 on DRST Spatial Performance on Day 1. (A-C) The increase in total span on day 1 at the middle dose was driven by increases on both repeated $\left({ }^{*} p=0.040\right)$ and non-repeated trials $\left({ }^{*} p=0.019\right)$. Error bars $=$ SEM.

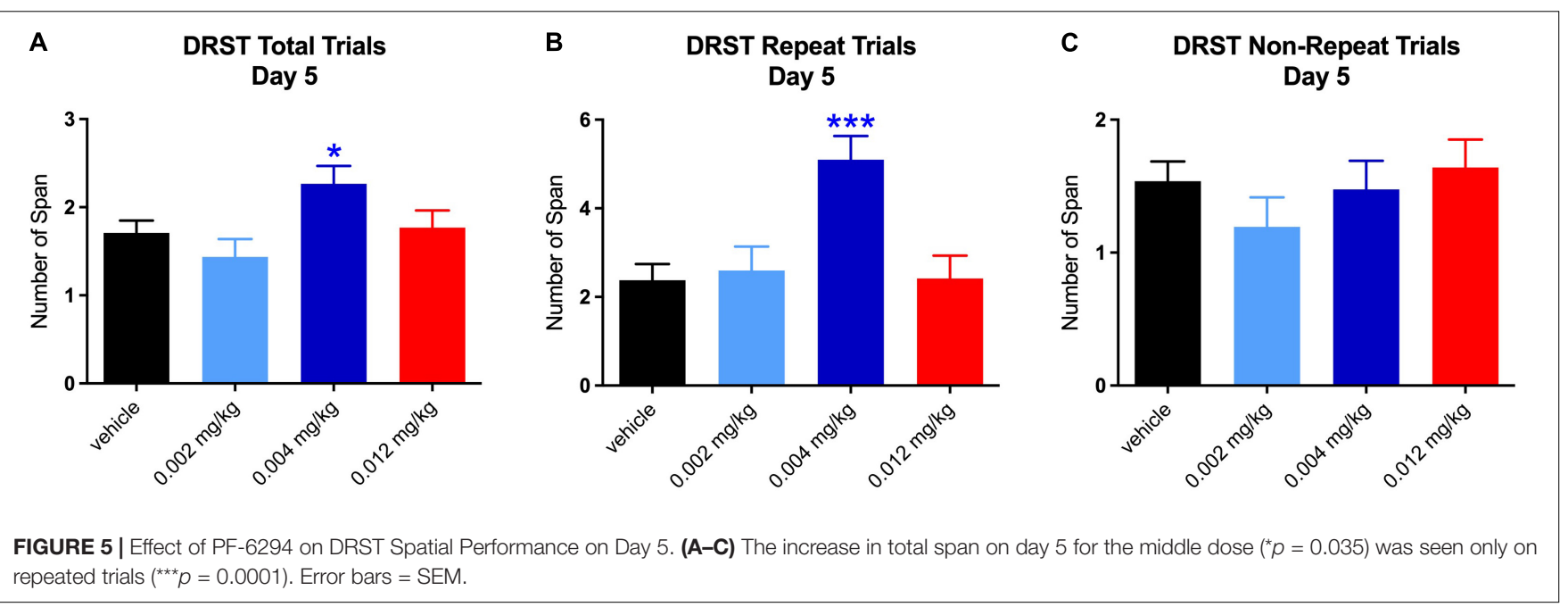

Funahashi, 2017) which is one of the first cortical regions to show age-related change. For example, magnetic resonance imaging studies have demonstrated that age-related atrophy is particularly prominent in the frontal lobes (Raz et al., 1997; Salat et al., 1999; Tisserand et al., 2002; Gunning-Dixon and Raz, 2003). Further, data from our laboratory and others over the past several decades has demonstrated an increase in neuroglial cells with dark cytoplasm and an increase in the amount of inclusion material in the neuroglial cells in the PFC, and a decrease in the thickness of layer 1 in area 46 of aged monkeys (Peters et al., 1994; Guttmann et al., 1998; Peters and Sethares, 2002; Makris et al., 2007; Wisco et al., 2008; Drag and Bieliauskas, 2010). In addition, degenerative changes in the myelin and underlying white matter occurs in the PFC of aged monkeys and correlates with cognitive performance (Peters et al., 1994). In addition, there is an increase in phagocytic and activated microglial in frontal white matter (Shobin et al., 2017), a loss of myelin integrity in area 46 as measured by decreased fractional anisotropy in PFC white matter (Makris et al., 2007), and an overall decrease in the volume of white matter with age (Wisco et al., 2008). Taken together, these findings show a particular vulnerability of the
PFC to age-related alterations in morphology that are related to declines in cognitive function.

\section{Dopamine, Prefrontal Cortex, and Aging}

The PFC is a main target of the mesocortical DA system and within this region dopamine is present in a higher concentration than in any other cortical region (Brown et al., 1975; Bannon and Roth, 1983; Felten and Sladek, 1983; Berridge et al., 1993). The PFC DA system is unique in being characterized by a high DA turnover rate, higher and more irregular neuronal firing, and a lack of autoreceptors (Bannon and Roth, 1983; Tam et al., 1990). Dopamine afferents in the human and non-human primate PFC have a bilaminar distribution, with the highest concentrations in layers I, II, III, and V, VI of the cortex. In terms of DA receptors, DA D1 receptors are approximately tenfold more concentrated in the PFC than the D2 receptors and are located primarily in the superficial layers of cortex (layer I, II, and IIIa) (Savasta et al., 1986; Goldman-Rakic et al., 1990, 1992; Lidow et al., 1991; Sawaguchi and Goldman-Rakic, 1991; Smiley et al., 1994). 
Neurochemical studies have demonstrated that dopamine (DA) plays a role in the cognitive functions subserved by the PFC (Brozoski et al., 1979; Sawaguchi and Goldman-Rakic, 1991; Goldman-Rakic et al., 1992; Arnsten et al., 1994; Castner and Goldman-Rakic, 2004). However, levels of DA are reduced in both aged monkeys and humans, with the most significant reductions occurring in the prefrontal and temporal cortices (Goldman-Rakic and Brown, 1981; Goldman-Rakic et al., 1990; Arnsten et al., 1994, 2015; Castner and Goldman-Rakic, 2004; Wang et al., 2019). In aged monkeys, DA levels in the PFC decrease by $56 \%$ (Goldman-Rakic and Brown, 1981; Arnsten et al., 1994) and there is evidence of a decrease in DA receptors in the brain regions including the PFC (Rinne et al., 1990, 1993; Suhara et al., 1991; Arnsten, 1993; Inoue et al., 2001; O’Brien et al., 2003). A study from our laboratory demonstrated no agerelated changes in post-synaptic D1 receptor binding in the PFC, which is in contrast to studies in the literature (Suhara et al., 1991; Arnsten, 1993). However, the sample size in our former study was small ( 8 young and 8 aged) and there was significant variability in binding density in the aged monkeys, both of which may have contributed to the lack of significant differences between age groups. Of particular interest though, we showed that there was a significant correlation between D1 receptor binding density and age-related impairment on the DRST task, with aged monkeys having the lowest spans on the DRST and D1 receptor binding density in dorsolateral PFC regions (Moore et al., 2005). This is particularily relevant, as the DRST task is the same task used in the present study on which aged monkeys demonstrated improved performance while receiving PF-6294. These promising findings build on similar studies in the field (Yohn et al., 2015; Wang et al., 2019) and provide further evidence of the efficacy of D1R agonists to effect the cognitive processes of the PFC in aging and perhaps disease processes that involve the PFC.

Further, it would be of interest in future studies to include young monkeys to determine the effect of non-catechol D1R agonists in younger monkeys as there are variable results in the literature. First, a recent study assessed the impact of a non-catechol D1R agonist similar to PF-6294 and demonstrated that young monkeys appeared to benefit from a low dose and aged monkeys benefited from a high dose (Williams et al., 2017). However, Arnsten et al. (1994) demonstrated that administration of the D1 antagonist, SCH-23390, to young monkeys caused impairments on a delayed response task but did not impair performance of aged monkeys. Therefore, future studies with PF-6294 should include young monkeys in order to determine its effect on a young brain not naturally depleted of DA.

\section{Dopamine Receptor Agonists and Aging}

Several studies have provided extensive evidence of a link between age related changes in dopamine receptor activity in various cortical regions, including the PFC, and performance on neuropsychological tests (Volkow et al., 2000; Kaasinen and Rinne, 2002; Wang et al., 2019) Arnsten et al. (1994) demonstrated that both a partial DA D1 agonist (SKF38393) and a full DA D1 agonist (dihydrexidine) improved performance by aged monkeys on the Delayed Response Task (DR - a working memory task). Further, the improved performance was reduced by the administration of the DA D1 antagonist, SCH23390. Similarly, Cai and Arnsten (1997), showed that the DA D1 full agonists, at low doses improved performance on the DR task which also was reversed by the DA D1 antagonist, SCH23390.

Yohn et al. (2015) demonstrated that the administration of DA D1 agonists, SKF38393, SKF81297 and A77636 reversed the negative effects of the DA D1 antagonist in rats while performing the T-Maze task. These findings reinforce the notion that the DA D1 receptors are functionally critical for successful completion of cognitive tasks.

Despite these extensive findings, a long-standing issue with the use of DA D1 agonists is that the selective pharmacological tools available are catechol-based molecules which are rapidly metabolized and desensitize the receptor quickly and therefore have a reduced effect over time. Recently, Gray et al. (2018), demonstrated a new class of selective, potent non-catechol DA D1 receptors agonists and partial agonists which are reported to have favorable in vivo pharmacokinetics, and reduced receptor desensitization. The D1-mediated pharmacology of compounds from this class were demonstrated in Wang et al. (2019) who showed that administration of the novel noncatechol D1R agonist, PF-3628, had an excitatory effect on working memory circuits in the PFC when directly applied via iontophoresis to aged rhesus monkeys. The administration of PF3628 produced an inverted- $U$ dose response curve, similar to the response observed in the current study, with increased firing of dorsolateral PFC neurons at low to moderated dose, especially in the oldest monkeys in the study. Of particular interest was the dorsolateral PFC neurons excited by PF-328 are in a cortical region involved in working memory. In the current study, PF6294 likely resulted in a similar restoration of excitatory action of DA neurons in the PFC and that could explain the improved performance on the DRST task. The dose response observed in these two studies adds to a body of evidence that cognitive benefits of D1 agonism may require an optimal level of dopamine system activation in order to enhance cognitive function and should continue to be explored.

\section{CONCLUSION}

The current study has demonstrated that the administration of the non-catechol DA D1 agonist, PF-6294, enhances performance on the DRST, a spatial working memory task, in aged rhesus monkeys. Though the significant effect was only observed on the 1st and last days of testing, these results are consistent with other recent studies demonstrating the efficacy of this class of non-catechol DA D1 agonists to have sustained efficacy (Wang et al., 2019). While this study utilized a small number of monkeys, the positive effect of the administration of the novel selective, potent, non-catechol DA D1 agonist PF-6294 adds to a growing body of literature supporting the therapeutic value of this class of agonists to slow or reverse age-related cognitive decline and warrants further study. 


\section{DATA AVAILABILITY STATEMENT}

The raw data supporting the conclusions of this article will be made available by the authors, without undue reservation.

\section{ETHICS STATEMENT}

The animal study was reviewed and approved by IACUC Boston University.

\section{AUTHOR CONTRIBUTIONS}

TM, RK, and RJK contributed to conception and design of the study. DY performed the statistical analysis. TM, RK, RJK,

\section{REFERENCES}

Albert, M. (1993). Neuropsychological and neurophysiological changes in healthy adult humans across the age range. Neurobiol. Aging 14, 623-625.

Alexander, G. E., Ryan, L., Bowers, D., Foster, T. C., Bizon, J. L., Geldmacher, D. S., et al. (2012). Characterizing cognitive aging in humans with links to animal models. Front. Aging Neurosci. 4:21. doi: 10.3389/fnagi.2012.00021

Arnsten, A. F. (1993). Catecholamine mechanisms in age-related cognitive decline. Neurobiol. Aging 14, 639-641. doi: 10.1016/0197-4580(93)90054-f

Arnsten, A. F., Cai, J. X., Murphy, B. L., and Goldman-Rakic, P. S. (1994). Dopamine D1 receptor mechanisms in the cognitive performance of young adult and aged monkeys. Psychopharmacology 116, 143-151.

Arnsten, A. F., Cai, J. X., Steere, J. C., and Goldman-Rakic, P. S. (1995). Dopamine D2 receptor mechanisms contribute to age-related cognitive decline: the effects of quinpirole on memory and motor performance in monkeys. J. Neurosci. 15(5 Pt 1), 3429-3439.

Arnsten, A. F., and Jentsch, J. D. (1997). The alpha-1 adrenergic agonist, cirazoline, impairs spatial working memory performance in aged monkeys. Pharmacol. Biochem. Behav. 58, 55-59.

Arnsten, A. F., Wang, M., and Paspalas, C. D. (2015). Dopamine's actions in primate prefrontal cortex: challenges for treating cognitive disorders. Pharmacol. Rev. 67, 681-696. doi: 10.1124/pr.115.010512

Arshad, M., Stanley, J. A., and Raz, N. (2016). Adult age differences in subcortical myelin content are consistent with protracted myelination and unrelated to diffusion tensor imaging indices. Neuroimage 143, 26-39. doi: 10.1016/j. neuroimage.2016.08.047

Bachevalier, J. (1993). Behavioral changes in aged rhesus monkeys. Neurobiol. Aging 14, 619-621.

Bäckman, L., Karlsson, S., Fischer, H., Karlsson, P., Brehmer, Y., Rieckmann, A., et al. (2011). Dopamine $\mathrm{D}(1)$ receptors and age differences in brain activation during working memory. Neurobiol. Aging 32, 1849-1856. doi: 10.1016/j. neurobiolaging.2009.10.018

Bäckman, L., Lindenberger, U., Li, S. C., and Nyberg, L. (2010). Linking cognitive aging to alterations in dopamine neurotransmitter functioning: recent data and future avenues. Neurosci. Biobehav. Rev. 34, 670-677. doi: 10.1016/j.neubiorev. 2009.12.008

Bäckman, L., Nyberg, L., Lindenberger, U., Li, S. C., and Farde, L. (2006). The correlative triad among aging, dopamine, and cognition: current status and future prospects. Neurosci. Biobehav. Rev. 30, 791-807. doi: 10.1016/j. neubiorev.2006.06.005

Bannon, M. J., and Roth, R. H. (1983). Pharmacology of mesocortical dopamine neurons. Pharmacol. Rev. 35, 53-68.

Beason-Held, L. L., Rosene, D. L., Killiany, R. J., and Moss, M. B. (1999). Hippocampal formation lesions produce memory impairment in the rhesus monkey. Hippocampus 9, 562-574. and DY wrote the manuscript. All authors contributed to manuscript review and revision and read and approved the submitted version.

\section{FUNDING}

This study was funded by Pfizer, Inc. and NIH/NIA RF1 AG043640.

\section{ACKNOWLEDGMENTS}

The authors would like to acknowledge the contributions of Rebecca Smith, Bethany Bowley, Penny Shultz, and Karen Slater for the invaluable assistance with this project.

Berridge, C. W., Arnsten, A. F., and Foote, S. L. (1993). Noradrenergic modulation of cognitive function: clinical implications of anatomical, electrophysiological and behavioural studies in animal models. Psychol. Med. 23, 557-564. doi: $10.1017 / \mathrm{s} 0033291700025332$

Bizon, J. L., Foster, T. C., Alexander, G. E., and Glisky, E. L. (2012). Characterizing cognitive aging of working memory and executive function in animal models. Front. Aging Neurosci. 4:19. doi: 10.3389/fnagi.2012.00019

Borella, E., Meneghetti, C., Ronconi, L., and De Beni, R. (2014). Spatial abilities across the adult life span. Dev. Psychol. 50, 384-392. doi: 10.1037/a0033818

Brown, R. M., Kehr, W., and Carlsson, A. (1975). Functional and biochemical aspects of catecholamine metabolism in brain under hypoxia. Brain Res. 85, 491-509.

Brozoski, T. J., Brown, R. M., Rosvold, H. E., and Goldman, P. S. (1979). Cognitive deficit caused by regional depletion of dopamine in prefrontal cortex of rhesus monkey. Science 205, 929-932.

Burke, C. J., Soutschek, A., Weber, S., Raja Beharelle, A., Fehr, E., Haker, H., et al. (2018). Dopamine receptor-specific contributions to the computation of value. Neuropsychopharmacology 43, 1415-1424. doi: 10.1038/npp.2017.302

Cai, J. X., and Arnsten, A. F. (1997). Dose-dependent effects of the dopamine D1 receptor agonists A77636 or SKF81297 on spatial working memory in aged monkeys. J. Pharmacol. Exp. Ther. 283, 183-189.

Castner, S. A., and Goldman-Rakic, P. S. (2004). Enhancement of working memory in aged monkeys by a sensitizing regimen of dopamine D1 receptor stimulation. J. Neurosci. 24, 1446-1450. doi: 10.1523/JNEUROSCI.3987-03.2004

Cohen, R. L., and Johansson, B. S. (1967). The activity trace in immediate memory: a re-evaluation. J. Verbal Learn. Verbal Behav. 6, 139-143. doi: 10.1016/S00225371(67)80064-1

Cotman, C. W., Head, E., Muggenburg, B. A., Zicker, S., and Milgram, N. W. (2002). Brain aging in the canine: a diet enriched in antioxidants reduces cognitive dysfunction. Neurobiol. Aging 23, 809-818.

Darusman, H. S., Call, J., Sajuthi, D., Schapiro, S. J., Gjedde, A., Kalliokoski, O., et al. (2014). Delayed response task performance as a function of age in cynomolgus monkeys (Macaca fascicularis). Primates 55, 259-267. doi: 10.1007/ s10329-013-0397-8

De Bundel, D., Femenía, T., DuPont, C. M., Konradsson-Geuken, Å, Feltmann, K., Schilström, B., et al. (2013). Hippocampal and prefrontal dopamine D1/5 receptor involvement in the memory-enhancing effect of reboxetine. Int. J. Neuropsychopharmacol. 16, 2041-2051. doi: 10.1017/S146114571300 0370

Di, L., Breen, C., Chambers, R., Eckley, S. T., Fricke, R., Ghosh, A., et al. (2017). Industry perspective on contemporary protein-binding methodologies: considerations for regulatory drug-drug interaction and related guidelines on highly bound drugs. J. Pharm. Sci. 106, 3442-3452. doi: 10.1016/j.xphs.2017.09. 005

Drag, L. L., and Bieliauskas, L. A. (2010). Contemporary review 2009: cognitive aging. J. Geriatr. Psychiatry Neurol. 23, 75-93. doi: 10.1177/0891988709358590 
Felten, D. L., and Sladek, J. R. Jr. (1983). Monoamine distribution in primate brain V. Monoaminergic nuclei: anatomy, pathways and local organization. Brain Res. Bull. 10, 171-284. doi: 10.1016/0361-9230(83)90045-x

Finch, C. E. (2009). The neurobiology of middle-age has arrived. Neurobiol. Aging 30, 515-520. doi: 10.1016/j.neurobiolaging.2008.11.011

Fischer, H., Nyberg, L., Karlsson, S., Karlsson, P., Brehmer, Y., Rieckmann, A., et al. (2010). Simulating neurocognitive aging: effects of a dopaminergic antagonist on brain activity during working memory. Biol. Psychiatry 67, 575-580. doi: 10.1016/j.biopsych.2009.12.013

Fisk, J. E., and Sharp, C. A. (2004). Age-related impairment in executive functioning: updating, inhibition, shifting, and access. J. Clin. Exp. Neuropsychol. 26, 874-890. doi: 10.1080/13803390490510680

Funahashi, S. (2017). Working memory in the prefrontal cortex. Brain Sci. 7:49. doi: 10.3390/brainsci7050049

Goldman-Rakic, P. S., and Brown, R. M. (1981). Regional changes of monoamines in cerebral cortex and subcortical structures of aging rhesus monkeys. Neuroscience 6, 177-187.

Goldman-Rakic, P. S., Lidow, M. S., and Gallager, D. W. (1990). Overlap of dopaminergic, adrenergic, and serotoninergic receptors and complementarity of their subtypes in primate prefrontal cortex. J. Neurosci. 10, 2125-2138. doi: 10.1523/jneurosci.10-07-02125.1990

Goldman-Rakic, P. S., Lidow, M. S., Smiley, J. F., and Williams, M. S. (1992). The anatomy of dopamine in monkey and human prefrontal cortex. J. Neural Transm. Suppl. 36, 163-177. doi: 10.1007/978-3-7091-9211-5_8

Gray, D. L., Allen, J. A., Mente, S., O’Connor, R. E., DeMarco, G. J., Efremov, I., et al. (2018). Impaired $\beta$-arrestin recruitment and reduced desensitization by non-catechol agonists of the D1 dopamine receptor. Nat. Commun. 9:674. doi: 10.1038/s41467-017-02776-7

Gunning-Dixon, F. M., and Raz, N. (2003). Neuroanatomical correlates of selected executive functions in middle-aged and older adults: a prospective MRI study. Neuropsychologia 41, 1929-1941.

Guttmann, C. R., Jolesz, F. A., Kikinis, R., Killiany, R. J., Moss, M. B., Sandor, T., et al. (1998). White matter changes with normal aging. Neurology 50, 972-978.

Herndon, J. G., Moss, M. B., Rosene, D. L., and Killiany, R. J. (1997). Patterns of cognitive decline in aged rhesus monkeys. Behav. Brain Res. 87, 25-34. doi: 10.1016/s0166-4328(96)02256-5

Inoue, M., Suhara, T., Sudo, Y., Okubo, Y., Yasuno, F., Kishimoto, T., et al. (2001). Age-related reduction of extrastriatal dopamine D2 receptor measured by PET. Life Sci. 69, 1079-1084. doi: 10.1016/s0024-3205(01)01205-x

Johnson, S. A., Sacks, P. K., Turner, S. M., Gaynor, L. S., Ormerod, B. K., Maurer, A. P., et al. (2016). Discrimination performance in aging is vulnerable to interference and dissociable from spatial memory. Learn. Mem. 23, 339-348. doi: $10.1101 / \mathrm{lm} .042069 .116$

Kaasinen, V., and Rinne, J. O. (2002). Functional imaging studies of dopamine system and cognition in normal aging and Parkinson's disease. Neurosci. Biobehav. Rev. 26, 785-793. doi: 10.1016/s0149-7634(02) 00065-9

Karlsson, S., Rieckmann, A., Karlsson, P., Farde, L., Nyberg, L., and Bäckman, L. (2011). Relationship of dopamine D1 receptor binding in striatal and extrastriatal regions to cognitive functioning in healthy humans. Neuroimage 57, 346-351. doi: 10.1016/j.neuroimage.2011.04.047

Kozak, R., Kiss, T., Dlugolenski, K., Johnson, D. E., Gorczyca, R. R., Kuszpit, K., et al. (2020). Characterization of PF-6142, a novel, non-catecholamine dopamine receptor D1 agonist, in murine and nonhuman primate models of dopaminergic activation. Front. Pharmacol. 11:1005. doi: 10.3389/fphar.2020. 01005

Kwon, D., Maillet, D., Pasvanis, S., Ankudowich, E., Grady, C. L., and Rajah, M. N. (2016). Context memory decline in middle aged adults is related to changes in prefrontal cortex function. Cereb. Cortex 26, 2440-2460. doi: 10.1093/cercor/ bhv068

Lai, Z. C., Moss, M. B., Killiany, R. J., Rosene, D. L., and Herndon, J. G. (1995). Executive system dysfunction in the aged monkey: spatial and object reversal learning. Neurobiol. Aging 16, 947-954.

Lidow, M. S., Goldman-Rakic, P. S., Gallager, D. W., and Rakic, P. (1991). Distribution of dopaminergic receptors in the primate cerebral cortex: quantitative autoradiographic analysis using $[3 \mathrm{H}]$ raclopride, $[3 \mathrm{H}]$ spiperone and [3H]SCH23390. Neuroscience 40, 657-671. doi: 10.1016/0306-4522(91) 90003-7

Makris, N., Papadimitriou, G. M., van der Kouwe, A., Kennedy, D. N., Hodge, S. M., Dale, A. M., et al. (2007). Frontal connections and cognitive changes in normal aging rhesus monkeys: a DTI study. Neurobiol. Aging 28, 1556-1567. doi: 10.1016/j.neurobiolaging.2006.07.005

McEwen, B. S., and Morrison, J. H. (2013). The brain on stress: vulnerability and plasticity of the prefrontal cortex over the life course. Neuron 79, 16-29. doi: 10.1016/j.neuron.2013.06.028

Mishkin, M. (1957). Effects of small frontal lesions on delayed alternation in monkeys. J. Neurophysiol. 20, 615-622.

Mishkin, M., and Pribram, K. H. (1956). Analysis of the effects of frontal lesions in monkey. II. Variations of delayed response. J. Comp. Physiol. Psychol. 49, 36-40.

Moore, T. L., Killiany, R. J., Herndon, J. G., Rosene, D. L., and Moss, M. B. (2003). Impairment in abstraction and set shifting in aged rhesus monkeys. Neurobiol. Aging 24, 125-134.

Moore, T. L., Killiany, R. J., Herndon, J. G., Rosene, D. L., and Moss, M. B. (2006). Executive system dysfunction occurs as early as middle-age in the rhesus monkey. Neurobiol. Aging 27, 1484-1493. doi: 10.1016/j.neurobiolaging.2005. 08.004

Moore, T. L., Schettler, S. P., Killiany, R. J., Herndon, J. G., Luebke, J. I., Moss, M. B., et al. (2005). Cognitive impairment in aged rhesus monkeys associated with monoamine receptors in the prefrontal cortex. Behav. Brain Res. 160, 208-221. doi: 10.1016/j.bbr.2004.12.003

Moore, T. L., Schettler, S. P., Killiany, R. J., Rosene, D. L., and Moss, M. B. (2009). Effects on executive function following damage to the prefrontal cortex in the rhesus monkey (Macaca mulatta). Behav. Neurosci. 123, 231-241. doi: 10.1037/ a0014723

Morcom, A. M., Bullmore, E. T., Huppert, F. A., Lennox, B., Praseedom, A., Linnington, H., et al. (2010). Memory encoding and dopamine in the aging brain: a psychopharmacological neuroimaging study. Cereb. Cortex 20, 743757. doi: 10.1093/cercor/bhp139

Müller, U., von Cramon, D. Y., and Pollmann, S. (1998). D1- versus D2-receptor modulation of visuospatial working memory in humans. J. Neurosci. 18, 27202728.

Murman, D. L. (2015). The impact of age on cognition. Semin. Hear. 36, 111-121. doi: 10.1055/s-0035-1555115

O’Brien, S., Rosene, D. L., and Luebke, J. I. (2003). GABAA receptor-mediated neurotransmission in the dentate gyrus of the rhesus monkey: comparison with the rat. Synapse 49, 287-289. doi: 10.1002/syn.10237

Peters, A., Leahu, D., Moss, M. B., and McNally, K. J. (1994). The effects of aging on area 46 of the frontal cortex of the rhesus monkey. Cereb. Cortex 4, 621-635.

Peters, A., and Sethares, C. (2002). Aging and the myelinated fibers in prefrontal cortex and corpus callosum of the monkey. J. Comp. Neurol. 442, 277-291.

Rapp, P. R., and Amaral, D. G. (1989). Evidence for task-dependent memory dysfunction in the aged monkey. J. Neurosci. 9, 3568-3576.

Rapp, P. R., and Amaral, D. G. (1991). Recognition memory deficits in a subpopulation of aged monkeys resemble the effects of medial temporal lobe damage. Neurobiol. Aging 12, 481-486.

Raz, N., Gunning, F. M., Head, D., Dupuis, J. H., McQuain, J., Briggs, S. D., et al. (1997). Selective aging of the human cerebral cortex observed in vivo: differential vulnerability of the prefrontal gray matter. Cereb. Cortex 7, 268282.

Rieckmann, A., Karlsson, S., Karlsson, P., Brehmer, Y., Fischer, H., Farde, L., et al. (2011). Dopamine D1 receptor associations within and between dopaminergic pathways in younger and elderly adults: links to cognitive performance. Cereb. Cortex 21, 2023-2032. doi: 10.1093/cercor/bhq266

Rinne, J. O., Hietala, J., Ruotsalainen, U., Säkö, E., Laihinen, A., Någren, K., et al. (1993). Decrease in human striatal dopamine D2 receptor density with age: a PET study with [11C]raclopride. J. Cereb. Blood Flow Metab. 13, 310-314. doi: 10.1038/jcbfm.1993.39

Rinne, J. O., Lönnberg, P., and Marjamäki, P. (1990). Age-dependent decline in human brain dopamine D1 and D2 receptors. Brain Res. 508, 349-352. doi: 10.1016/0006-8993(90)90423-9

Rosvold, H. E., Szwarcbart, M. K., Mirsky, A. F., and Mishkin, M. (1961). The effect of frontal-lobe damage on delayed response performance in chimpanzees. J. Comp. Physiol. Psychol. 54, 368-374. 
Salat, D. H., Kaye, J. A., and Janowsky, J. S. (1999). Prefrontal gray and white matter volumes in healthy aging and Alzheimer disease. Arch. Neurol. 56, 338-344.

Salthouse, T. A. (2016). Continuity of cognitive change across adulthood. Psychon. Bull. Rev. 23, 932-939. doi: 10.3758/s13423-015-0910-8

Savasta, M., Dubois, A., and Scatton, B. (1986). Autoradiographic localization of D1 dopamine receptors in the rat brain with $[3 \mathrm{H}] \mathrm{SCH} 23390$. Brain Res. 375, 291-301. doi: 10.1016/0006-8993(86)90749-3

Sawaguchi, T., and Goldman-Rakic, P. S. (1991). D1 dopamine receptors in prefrontal cortex: involvement in working memory. Science 251, 947-950.

Sawaguchi, T., Matsumura, M., and Kubota, K. (1990). Effects of dopamine antagonists on neuronal activity related to a delayed response task in monkey prefrontal cortex. J. Neurophysiol. 63, 1401-1412.

Shobin, E., Bowley, M. P., Estrada, L. I., Heyworth, N. C., Orczykowski, M. E., Eldridge, S. A., et al. (2017). Microglia activation and phagocytosis: relationship with aging and cognitive impairment in the rhesus monkey. Geroscience 39, 199-220. doi: 10.1007/s11357-017-9965-y

Smiley, J. F., Levey, A. I., Ciliax, B. J., and Goldman-Rakic, P. S. (1994). D1 dopamine receptor immunoreactivity in human and monkey cerebral cortex: predominant and extrasynaptic localization in dendritic spines. Proc. Natl. Acad. Sci. U.S.A. 91, 5720-5724. doi: 10.1073/pnas.91.12.5720

Soutschek, A., Gvozdanovic, G., Kozak, R., Duvvuri, S., de Martinis, N., Harel, B., et al. (2020a). Dopaminergic D1 receptor stimulation affects effort and risk preferences. Biol. Psychiatry 87, 678-685. doi: 10.1016/j.biopsych.2019.09.002

Soutschek, A., Kozak, R., de Martinis, N., Howe, W., Burke, C. J., Fehr, E., et al. (2020b). Activation of D1 receptors affects human reactivity and flexibility to valued cues. Neuropsychopharmacology 45, 780-785. doi: 10.1038/s41386-0200617-z

Suhara, T., Fukuda, H., Inoue, O., Itoh, T., Suzuki, K., Yamasaki, T., et al. (1991). Age-related changes in human D1 dopamine receptors measured by positron emission tomography. Psychopharmacology 103, 41-45. doi: 10.1007/ bf02244071

Szmalec, A., Duyck, W., Vandierendonck, A., Mata, A. B., and Page, M. P. (2009). The Hebb repetition effect as a laboratory analogue of novel word learning. Q. J. Exp. Psychol. 62, 435-443. doi: 10.1080/17470210802386375

Takahashi, H. (2013). PET neuroimaging of extrastriatal dopamine receptors and prefrontal cortex functions. J. Physiol. 107, 503-509. doi: 10.1016/j.jphysparis. 2013.07.001

Tam, S. Y., Elsworth, J. D., Bradberry, C. W., and Roth, R. H. (1990). Mesocortical dopamine neurons: high basal firing frequency predicts tyrosine dependence of dopamine synthesis. J. Neural Transm. Gen. Sect. 81, 97-110. doi: 10.1007/ bf01245830

Thierry, A. M., Pirot, S., Gioanni, Y., and Glowinski, J. (1998). Dopamine function in the prefrontal cortex. Adv. Pharmacol. 42, 717-720.

Tisserand, D. J., Pruessner, J. C., Sanz Arigita, E. J., van Boxtel, M. P., Evans, A. C., Jolles, J., et al. (2002). Regional frontal cortical volumes decrease differentially in aging: an MRI study to compare volumetric approaches and voxel-based morphometry. Neuroimage 17, 657-669.

Toepper, M., Markowitsch, H. J., Gebhardt, H., Beblo, T., Bauer, E., Woermann, F. G., et al. (2014). The impact of age on prefrontal cortex integrity during spatial working memory retrieval. Neuropsychologia 59, 157-168. doi: 10.1016/ j.neuropsychologia.2014.04.020

Volkow, N. D., Ding, Y. S., Fowler, J. S., Wang, G. J., Logan, J., Gatley, S. J., et al. (1996). Dopamine transporters decrease with age. J. Nucl. Med. 37, 554-559.

Volkow, N. D., Gur, R. C., Wang, G. J., Fowler, J. S., Moberg, P. J., Ding, Y. S., et al. (1998). Association between decline in brain dopamine activity with age and cognitive and motor impairment in healthy individuals. Am. J. Psychiatry 155, 344-349. doi: 10.1176/ajp.155.3.344

Volkow, N. D., Logan, J., Fowler, J. S., Wang, G. J., Gur, R. C., Wong, C., et al. (2000). Association between age-related decline in brain dopamine activity and impairment in frontal and cingulate metabolism. Am. J. Psychiatry 157, 75-80. doi: 10.1176/ajp.157.1.75

Wang, M., Datta, D., Enwright, J., Galvin, V., Yang, S. T., Paspalas, C., et al. (2019). A novel dopamine D1 receptor agonist excites delay-dependent working memory-related neuronal firing in primate dorsolateral prefrontal cortex. Neuropharmacology 150, 46-58. doi: 10.1016/j.neuropharm.2019. 03.001

Wang, Y., Chan, G. L., Holden, J. E., Dobko, T., Mak, E., Schulzer, M., et al. (1998). Age-dependent decline of dopamine D1 receptors in human brain: a PET study. Synapse 30, 56-61.

Wass, C., Pizzo, A., Sauce, B., Kawasumi, Y., Sturzoiu, T., Ree, F., et al. (2013). Dopamine D1 sensitivity in the prefrontal cortex predicts general cognitive abilities and is modulated by working memory training. Learn. Mem. 20, 617-627. doi: 10.1101/lm.031971.113

Williams, G., Kozak, R., Abbot, A., Fonseca, K., Schmidt, C., Gray, D., et al. (2017). Novel non-catechol dopamine D1 receptor agonist pretreatment protects against the cognitive impairment induced by increased memory load in nonhuman primates. Soc. Neurosci. 29.

Wisco, J. J., Killiany, R. J., Guttmann, C. R., Warfield, S. K., Moss, M. B., and Rosene, D. L. (2008). An MRI study of age-related white and gray matter volume changes in the rhesus monkey. Neurobiol. Aging 29, 1563-1575. doi: 10.1016/j.neurobiolaging.2007.03.022

Yohn, S. E., Santerre, J. L., Nunes, E. J., Kozak, R., Podurgiel, S. J., Correa, M., et al. (2015). The role of dopamine D1 receptor transmission in effort-related choice behavior: effects of D1 agonists. Pharmacol. Biochem. Behav. 135, 217-226. doi: 10.1016/j.pbb.2015.05.003

Young, D., Popiolek, M., Trapa, P., Fonseca, K. R., Brevard, J., Gray, D. L., et al. (2020). D1 agonist improved movement of parkinsonian nonhuman primates with limited dyskinesia side effects. ACS Chem. Neurosci. 11, 560-566. doi: 10.1021/acschemneuro.9b00589

Conflict of Interest: DY, KF, DV, DG, RB-G, and RK were employed by Pfizer, Inc., United States.

The remaining authors declare that the research was conducted in the absence of any commercial or financial relationships that could be construed as a potential conflict of interest.

Publisher's Note: All claims expressed in this article are solely those of the authors and do not necessarily represent those of their affiliated organizations, or those of the publisher, the editors and the reviewers. Any product that may be evaluated in this article, or claim that may be made by its manufacturer, is not guaranteed or endorsed by the publisher.

Copyright (c) 2021 Moore, Young, Killiany, Fonseca, Volfson, Gray, Balice-Gordon and Kozak. This is an open-access article distributed under the terms of the Creative Commons Attribution License (CC BY). The use, distribution or reproduction in other forums is permitted, provided the original author(s) and the copyright owner(s) are credited and that the original publication in this journal is cited, in accordance with accepted academic practice. No use, distribution or reproduction is permitted which does not comply with these terms. 